\title{
A comparison of pre-emptive with preventive epidural analgesia in the patients undergoing major gynecologic surgery
}

\author{
Simin Atashkhoyi ${ }^{\star}$, Mehri Jafari Shobeiri, Alireza Azari Kalejahi and Pouya Hatami Marandi \\ Department of Anesthesiology, Women's Reproductive Health Research Center, Tabriz University of Medical Sciences, \\ Tabriz, Iran. \\ Accepted 4 July, 2012
}

\begin{abstract}
Pain management is a crucial component in the care of the postoperative patient. Although pre-emptive analgesia is commonly used for the management of postoperative pain, timing the analgesic administration is unclear. This study was designed to compare the efficacy of pre-emptive epidural analgesia (EA) with preventive EA in the patients undergoing major gynecologic surgery. A randomized, double-blinded trial was performed in 50 women of physical status American Society of Anesthesiologists (ASA) 1-3 undergoing major gynecologic surgery. Prior to induction of general anesthesia an epidural catheter was inserted in the patients of the two groups. Patients were allocated randomly into one of two groups; pre-emptive group $(n=25)$ received $12 \mathrm{ml}$ of $0.125 \%$ bupivacaine and $50 \mu \mathrm{g}$ fentanyl epidurally $20 \mathrm{~min}$ before the incision of surgery and the preventive group $(n=25)$ received the same of agents $\mathbf{2 0}$ min before the end of surgery via the epidural catheter. Preventive compared to pre-emptive EA had a significantly increased interval between the analgesic requests $(P<0.001)$. The preventive group compared to the pre-emptive group had significantly decreased postoperative visual analog scale (VAS) in post anesthesia care unit (PACU) and up to $3 \mathrm{~h}$ after surgery $(P<0.001)$. A preventive EA before the end of operation provides an improved postoperative analgesia in comparison to pre-emptive EA with no side effects in patients undergoing major gynecologic surgery.
\end{abstract}

Key words: Gynecologic surgery, postoperative pain, epidural administration, pre-emptive analgesia, bupivacaine, fentanyl.

\section{INTRODUCTION}

Peripheral and centraı nervous system sensitization results in postoperative pain hypersensitivity at the incision site and in surrounding tissues (Updike et al., 2003; Farouk, 2008). "Pre-emptive" analgesia describes the concept of being able to reduce pain perception and overall analgesic needs by using agents to inhibit central nervous system sensitization before the application of painful stimuli (Akural et al., 2002; Hony et al., 2008; Atashkhoyi et al., 2011). "Preventive" analgesia includes any peri-operative analgesic regimen that is able to control pain-induced sensitization of the central nervous system (CNS), hence

*Corresponding author. E-mail: satashkhoyi@gmail.com. reducing both the development and the persistence of pathologic pain (Lavand'hmme et al., 2005).

There are studies concerning different routes of preemptive analgesia, such as wound infiltration, intravenous, intrathecally or epidurally, that improve anesthetic and analgesic quality (Shapiro et al., 2003; Wilson et al., 2008; Bong et al., 2005). Superior analgesia after different operations was achieved with epidural injection of analgesics compared to other routes of analgesic administration (Zutshi et al., 2005; Klasen et al., 2005; Wu et al., 2000). The timing of epidural analgesic administration (pre-incision, during operation, at emergence from anesthesia or after operation) is also another impact factor of the efficacy of analgesia. Richards et al. (1998) was unable to detect any significant difference in either of 
the outcome of pain measures for the two groups that received epidural local anesthetic with combination of opioid 15 min prior to surgical incision or 15 min prior to skin closure. Katz et al. (2004) showed the short term beneficial effects of preventive epidural analgesia in patients undergoing gynecologic laparotomy. Another study showed that fentanyl administered epidurally before awaking at the end of the elective abdominal surgery versus prior to surgical incision produce lower pain scores (Esmaoglu et al., 2001).

In this study, we compared the epidural administration of local anesthetic with combination of fentanyl before the surgical incision (pre-emptive analgesia) or at the end of surgery (preventive analgesia) in reducing the postoperative pain following major gynecologic surgery.

\section{MATERIALS AND METHODS}

Fifty female patients were enrolled in this prospective, randomized, and double-blind clinical trial, with approval from the medical Ethics Committee and written consent obtained from the participants. The inclusion criteria were physical status American Society of Anesthesiologists (ASA) I-III, aged between 20 and 80 years undergoing elective major gynecologic surgery. Exclusion criteria were contraindication for epidural puncture, known allergy to local anesthetics, the patients consumed opioids and non-steroidal antiinflammatory drugs (NSAIDs) preoperatively, and patients with physiological and psychological disorders.

Patients were pre-medicated with oral $5 \mathrm{mg}$ diazepam $1 \mathrm{~h}$ before operation. Before the induction of anesthesia, an epidural catheter was placed at the $L_{3-4}$ intervertebral space in the patients. Patients were allocated randomly into one of two groups; patients in the preemptive group $(n=25)$ received $12 \mathrm{ml}$ of $0.125 \%$ bupivacaine (Bupivacaine mylan, Delpharm, France) and $50 \mu \mathrm{g}$ fentanyl (Aburaihan Pharmaceutical, Iran) epidurally $20 \mathrm{~min}$ before the incision of surgery. Patients in preventive group $(n=25)$ received the same agents 20 min before the end of surgery via the epidural catheter. General anesthesia (GA) was induced in all patients with fentanyl 1 - $2 \mu \mathrm{g} / \mathrm{kg}$, thiopentone $4 \mathrm{mg} / \mathrm{kg}$, and succynilcoline 0.6 $\mathrm{mg} / \mathrm{kg}$ was used to facilitate tracheal intubation. Anesthesia was maintained with nitrous oxide $\left(\mathrm{N}_{2} \mathrm{O}\right) 50 \%$ in oxygen $\left(\mathrm{O}_{2}\right)$, isoflurane 0.5 - 1 minimum alveolar concentration (MAC), and pancronium $0.05 \mathrm{mg} / \mathrm{kg}$. Fentanyl (1 $\mu \mathrm{g} / \mathrm{kg}$ intravenous (i.v.)) was used as supplement of analgesia during surgery, if necessary. At the end of surgery, patients were transferred to post anesthesia care unit (PACU) and then referred to the intensive care unit (ICU). All patients received patient-controlled epidural analgesia (PCEA) with $0.125 \%$ of bupivacaine $(1.25 \mathrm{mg} / \mathrm{ml})$ and $5 \mu \mathrm{g} / \mathrm{ml}$ fentanyl delivered with a patient controlled analgesia (PCA) device (Abbott Pain Management Provider; Abbott Laboratories, North Chicago, IL) for $24 \mathrm{~h}$ postoperatively. No background infusion was used in either group. PCA pump was programmed to deliver $2 \mathrm{ml}$ bolus with a lockout interval of $15 \mathrm{~min}$ in both groups. If analgesia was inadequate and visual analogue scale (VAS) >4 for $30 \mathrm{~min}$, meperidine $(0.5-1 \mathrm{mg} / \mathrm{kg}$ every $6 \mathrm{~h})$ was given intramuscularly as rescue measure of pain management.

Intraoperative monitoring consisted of electrocardiogram (ECG), pulse oximetry, end tidal $\mathrm{CO}_{2}$ concentration $\left(\mathrm{ETCO}_{2}\right)$, urine output and hemodynamic variables. Hydration was achieved with lactated Ringer's solution. Three surgeons performed the surgical procedures, and the anesthesia was provided by two anesthesiologists who were responsible for monitoring of anesthesia and postoperative analgesia management. The surgeons, second anesthesiologist (Azari A) and patients were blinded to the study.
All study-related measurements were taken by the same anesthesiologist who was not aware of the treatment allocation of the patients. Pain scores on a visual analogue scale (VAS; $0=$ no pain, $10=$ worst pain imaginable) were recorded in the PACU (at emergence and during $24 \mathrm{~h}$ post-operatively). First request to analgesia, total analgesic consumption (epidurally or systemically administered drugs) and the presences of side effects (nauseavomiting, drowsiness, dizziness, and respiratory depression) were also recorded during the first $24 \mathrm{~h}$ after operation.

The sample size was based on a power calculation which showed that 50 patients were necessary to achieve $80 \%$ power to detect a prolongation of postoperative first analgesic demand of $3 \mathrm{~h}$ between patients treated with pre-emptive or preventive epidural analgesia with $\alpha=0.05$. Data were presented as mean $\pm S D$. Demographic data, dose of intra-operative fentanyl, duration of surgery and anesthesia, IV fluid were compared using the student $t$ test. Postoperative pain scores and incidence of side effects were compared by $x^{2}$-square test between two groups. A $p$ value $\leq 0.05$ was considered statistically significant.

\section{RESULTS}

There were no significant differences between the groups with regards to age, weight, height, duration of surgery, type of surgery, and ASA status of classification (Table 1). Moreover, there were no significant differences between the two groups regarding to intraoperative data such as intravenous fluid, urine output, and blood loss (Table 1). Intraoperative fentanyl consumption was lower in the pre-emptive group than those in the preventive group $(128.00 \pm 38.40$ vs. $158.00 \pm 37.30 \mu \mathrm{g} ; \mathrm{p}=0.007$, Table 1). The hemodynamic changes during anesthesia were presented in Figure 1. Decreased of mean arterial pressure (MAP) and heart rate (HR) occurred more often in the pre-emptive group than in the preventive group, although this difference was not significant.

Additionally, the VAS was significantly lower for the patients in the preventive group at emergence $(p<0.001)$, and up to 3 hours postoperatively $(p<0.001)$ than those in the pre-emptive group, but not at 6,12 , and $24 \mathrm{~h}$ after surgery (Table 2 and Figure 1). Table 2 shows that the time to first request for analgesics were significantly longer in the preventive group $(5.06 \pm 2.54$ vs. $2.44 \pm$ $1.58 \mathrm{~h} ; \mathrm{p}<0.001)$. The frequency of rescue epidural analgesia by using the PCEA was significantly lower in preventive group than those in the patients of preemptive group ( 7 in preventive group vs. 12 in preemptive group; $p<0.001)$. There were no differences in the consumption of additional analgesics (meperidine) between two groups. Also, there were no differences in hemodynamic or respiratory instability among patients of two groups during $24 \mathrm{~h}$ post operation. Other postoperative side effects also were absent in both groups. Furthermore, no patient experienced motor block during postoperative period.

\section{DISCUSSION}

The results of the present study indicate that epidural 
Table 1. Patient's characteristics and intraoperative variables in the two groups [number (\%) and mean $\pm S D$ ].

\begin{tabular}{|c|c|c|c|}
\hline Parameter $\quad$ Group & Pre-emptive group $(n=25)$ & Preventive group $(n=25)$ & p-value \\
\hline Age (years) & $56.24 \pm 10.45$ & $56.16 \pm 16.87$ & 0.98 \\
\hline Weight $(\mathrm{kg})$ & $73.12 \pm 15.91$ & $70.80 \pm 12.67$ & 0.57 \\
\hline Height(cm) & $159.36 \pm 07.33$ & $159.84 \pm 07.00$ & 0.81 \\
\hline ASA $(I / I I / I I I)$ & $12 / 6 / 7$ & $13 / 7 / 5$ & 0.42 \\
\hline Duration of surgery(min) & $171.00 \pm 46.02$ & $191.40 \pm 40.22$ & 0.10 \\
\hline \multicolumn{4}{|l|}{ Type of surgery (\%) } \\
\hline Lymphadenectomy & $15(60)$ & $16(64)$ & \\
\hline Abdominal hysterectomy & $8(32)$ & $7(28)$ & 0.76 \\
\hline Vaginal hysterectomy & $2(8)$ & 2(8) & \\
\hline Total fluid during anesthesia $(\mathrm{ml})$ & $3240.00 \pm 737.67$ & $3484.00 \pm 655.54$ & 0.22 \\
\hline Intraoperative blood loss (ml) & $304.00 \pm 113.57$ & $345.00 \pm 121.55$ & 0.87 \\
\hline Urine output (ml) & $594.00 \pm 323.01$ & $604.80 \pm 309.75$ & 0.6 \\
\hline Intraoperative fentanyl consumption $(\mu \mathrm{g})$ & $128.00 \pm 38.40$ & $158.00 \pm 37.30$ & 0.007 \\
\hline Intraoperative hypotension & 0 & 0 & 1.0 \\
\hline Intraoperative bradycardia & 0 & 0 & 1 \\
\hline
\end{tabular}

Table 2. Postoperative pain variables in two groups [number (\%) and mean $\pm S D$ ].

\begin{tabular}{lccc}
\hline \multicolumn{1}{c}{ Group } & Pre-emptive $(\mathbf{n}=\mathbf{2 5})$ group & Preventive $(\mathbf{n}=\mathbf{2 5})$ group & p-value \\
\hline Parameter & & & \\
Mean VAS score & $1.84 \pm 0.34$ & $0.16 \pm 0.07$ & $<0.001$ \\
in PACU & $4.44 \pm 0.30$ & $1.82 \pm 0.39$ & $<0.001$ \\
3 h later & $3.00 \pm 1.50$ & $2.78 \pm 1.62$ & 0.11 \\
6 h later & $2.16 \pm 1.14$ & $2.56 \pm 1.63$ & 0.32 \\
12 h later & $1.28 \pm 0.28$ & $1.24 \pm 0.29$ & 0.87 \\
24 h later & & & \\
& $2.44 \pm 1.58$ & $5.06 \pm 2.54$ & $<0.001$ \\
Time to first analgesic request (h) & 7 & 12 & $<0.001$ \\
Number of PCEA rescue & & & \\
\hline
\end{tabular}

injection of bupivacaine and fentanyl 20 min before the end of surgery (preventive) provided better analgesia, which was referred as lower pain scores, longer duration of the first analgesic request, and lower postoperative analgesic consumption during the first $24 \mathrm{~h}$ after major gynecologic surgery compared with pre-incisional (preemptive) epidural analgesia. Although some studies have shown that administration of local or regional anesthesia just minutes before surgical procedures seems to be ineffective for prevention of postoperative pain (Updike et al., 2003), most studies succeeded to show the benefits pre-emptive analgesia by intravenous or epidural analgesia (Farouk, 2008; Akural et al., 2002; Hony, 2008). Studies have also showed that long-lasting (on the day before operation) pre-emptive epidural analgesia with local anesthetic and opioid reduced postoperative consump- tion of analgesics with improvement pain rating $(\mathrm{Wu}$ et al., 2000; Richards et al., 1998) were unable to detect any significant difference in either of the postoperative pain outcomes for the pre-incisional epidural analgesia when it was compared with injection of epidural analgesics at the end of surgery. Others reported that analgesics administered epidurally prior awaking at the end of operation is more effective for postoperative pain control than those given before the induction of anesthesia (Lavand'hmme et al., 2005; Katz et al., 2004; Esmaoglu et al., 2001; Gottschalk et al., 2008).

The present study findings demonstrates that injection of analgesics epidurally prior the end of surgery effectively suppresses all possible nociceptor transduction pathways, preventing central sensitization and improving postoperative pain management. Epidural anesthesia 


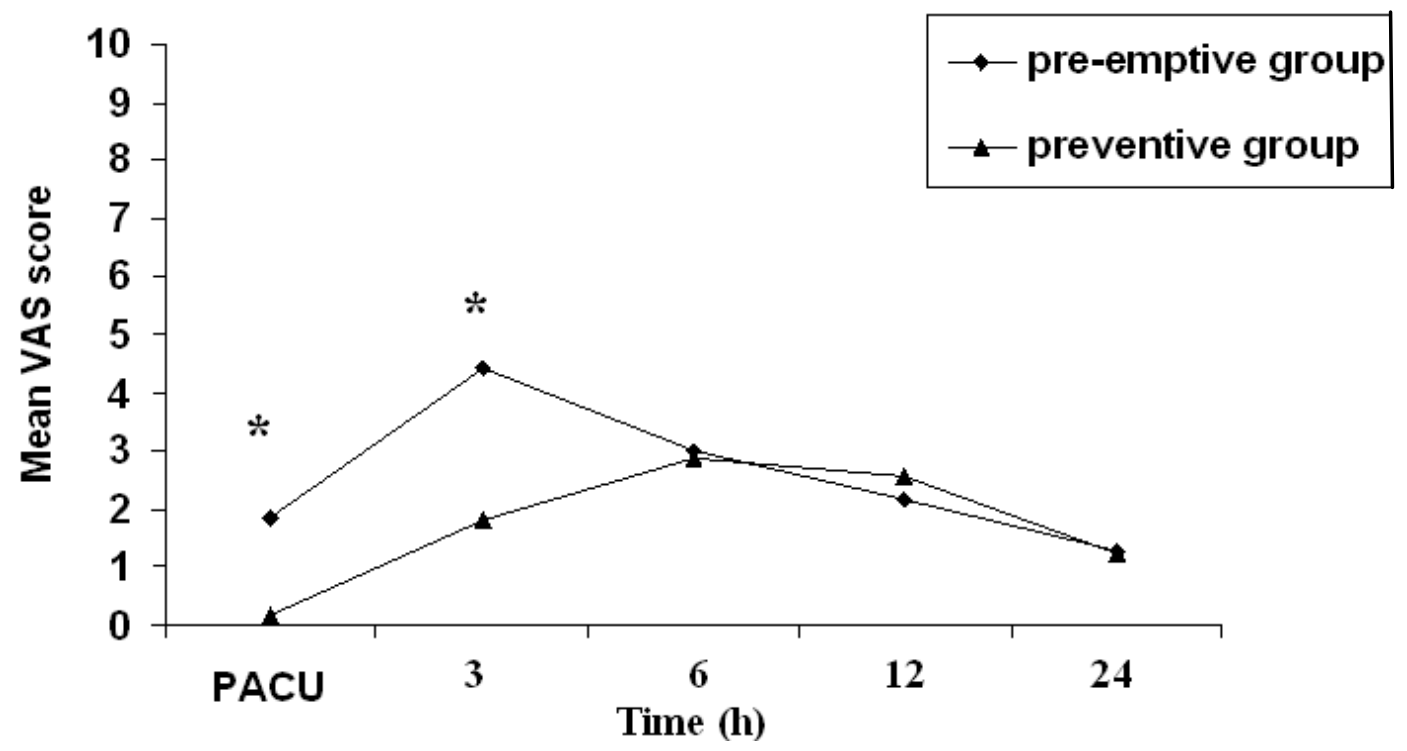

Figure 1. Visual analog scale (VAS) at each time interval of the study ( $\left.{ }^{*} p<0.001\right)$.

with bupivacaine blocks the sensory input of surgical stimuli (Wu et al., 2000). Fentanyl activates the opioid receptors and suppresses the initial response of dorsal horn nociceptive neurons and $\mathrm{C}$-fiber stimulation (Akural et al., 2002). One possible explanation for our findings is that administration of opioids as premedication or during induction and maintenance of anesthesia may provide pre-emptive analgesia (Bong CL et al., 2005).

There are a number of limitations to this study: First, the study limited assessment of postoperative analgesia to the first 24 postoperative hours. Secondly, the study was not large enough to assess safety. Thirdly, we did not control group (epidural injection of placebo as preemptive and preventive), and the highest reported pain score was below 5 in this study (Table 2).

\section{Conclusion}

A preventive epidural analgesia with bupivacaine and fentanyl before the end of operation provides an improved postoperative analgesia in comparison to preemptive epidural injection of same agents with no side effects in patients undergoing major gynecologic surgery. Further trials are necessary to evaluate preventive analgesia on chronic postoperative pain.

\section{REFERENCES}

Updike GM, Manolitsas TP, Cohn DE, Eaton LA, Fowler JM, Young DC, Copeland LJ (2003). Pre-emptive analgesia in gynecologic surgical procedures: preoperative wound infiltration with ropivacaine in patients who undergo laparotomy through a midline vertical incision. Am. J. Obstet. Gynenecol. 188:901-905.

Farouk S (2008). Pre-incisional epidural magnesium provides pre-emptive and preventive analgesia in patients undergoing abdominal hysterectomy, Br. J. Anesth. 101(5):694-699.

Akural El, Salomaki TE, Tekay AH, Bloigu AH, Alahuhta SM (2002). Pre-emptive effect of epidural sufentanil in abdominal hysterectomy. Br. J. Anesth. 88(6):803-808.

Atashkhoyi S, Azarfarin R, Mehrzad Sadagiani M (2011). Efficacy of pre-incisional subcutaneous infiltration of low-dose ketamine on postoperative pain after cesarean section. Pak. J. Med. Sci. 27(2):344-347.

Hony JY, Kyung TL (2008). Effect of pre-emptive epidural analgesia on cytokine response and postoperative pain in laparoscopic radical hysterectomy for cervical cancer. American Society of Reg. Anesth. Pain Med. 33(1):44-51.

Lavand'hmme P, Kock DM, Waterlosshilde RN (2005). Intra-operative Epidural Analgesia Combined With Ketamine Provides Effective Preventive Analgesia in Patients Undergoing Major Digestive Surgery. Anesthesiol. 103(4):813-820.

Shapiro A, Zohar E, Hoppenstein D, Nisim If, Jedeikin R, Fredman B (2003). A Comparison of Three Techniques for Acute Postoperative Pain Control Following Major Abdominal Surgery. J. Clin. Anesth. 15(5):345-350.

Wilson JA, Nimmo AF, Fleel wood-walker SM, Colvin LA (2008). A randomized double- blind trial of the effect of pre-emptive epidural ketamine on persistent pain after lower limb amputation. International Association for the Study of Pain 135(1-2):108-118.

Bong CL, Samuel M, Ng JM, Iq-Yam C (2005). Effect of Pre- emptive Epidural Analgesia on Post-thoracotomy Pain. J. Cardiothorac. Vasc. Anesth. 19(6):786-793.

Zutshi M, Delaney CP, Senagore AJ, Mikhail N, Lewis BDO, Jason T, Conor MS , Victor W, Fazio MD (2005). Randomized controlled trial comparing the controlled rehabilitation with early ambulation and diet pathway versus the controlled rehabilitation with early ambulation and diet with preemptive epidural anesthesia/anesthesia after laparatomy and intestinal resection. Am. J. Surg. 189(3):268-272.

Klasen J, Haas M, Graf S, Harbach H, Quinzio L, Jurgensen I, Hempelmann $G$ (2005). Impact on postoperative pain of long-lasting pre-emptive epidural analgesia before total hip replacement: a prospective, randomized, double-blind study. Anesth. 60(2):118-123.

Wu CT, Yeh CC, Yu JC, Lee MMS, Tao PL, Ho ST, Wong CS (2000). Pre-incisional epidural ketamine, morphine and bupivacaine combined with epidural and general anesthesia provides pre-emptive analgesia for upper abdominal surgery. Acta Anesthesiol. Scandinavia 44(1):63-68.

Richards JT, Read JRM, Chambers WA (1998). Epidural anesthesia as a method of pre-emptive analgesia for abdominal hysterectomy. 
Anesth. 53(3):296-307.

Katz J, Cohen L (2004). Preventive Analgesia Is Associated With Reduced Pain Disability 3 Weeks but Not 6 Months after Major Gynecologic Surgery by Laparotomy. Anesthesiol. 101(1):169-174.

Esmaoglu A, Cuha Y, Boyaci A (2001). Pre-emptive efficacy of epidural fentanyl in elective abdominal surgery. Eur. J. Anesthesiol. 18(1):59-
Gottschalk A, Freitag M, Steinacker E, Krei S, Remp C, Staude HJ, Strate T, Stand T (2008). Pre-incisional epidural ropivacaine, sufentanil, clonidine, and (S) 1-ketamine do not provide pre-emptive analgesia in patients undergoing major pancreatic surgery. $\mathrm{Br}$. J. Anaesth. 100(1):36-41. 